azithromycin resistance it is highly recommended to determine the resistance pattern of the respective gonococcal strain by culture performance.

\section{P2.097 PCR FOR DIRECT DETECTION OF THE MOSAIC NEISSERIA GONORRHOEAE PENA GENE IN URINES AND CERVICAL, RECTAL AND TONSILLAR SWABS}

doi:10.1136/sextrans-2013-051184.0361

1,2A P Van Dam, 'L Thiel, 'M Dierdorp, 'S Bruisten. 'Public Health Laboratory, Cluster Infectious Diseases, Municipal Health Service, Amsterdam, The Netherlands; ${ }^{2}$ Onze Lieve Vrouwe Gasthuis, dept of Medical Microbiology, Amsterdam, The Netherlands

Introduction The mosaic penA gene, partly derived from commensal Neisseria strains, is strongly associated with diminished susceptibility of Neisseria gonorhoeae ( $\mathrm{Ng}$ ) against cephalosporins. We developed a direct PCR test for Ng-positive clinical specimens to detect the mosaic penA gene.

Methods Swabs and urines from patients with gonorrhoea were in medium for NAAT testing (Aptima Combo 2). Corresponding Ng strains were obtained by culture on selective GC agar plates and stored at $-80^{\circ} \mathrm{C}$. Presence of a mosaic penA gene in these strains was demonstrated by PCR.

Results Using one conserved forward primer and two reverse primers, specific for mosaic- and wild type PenA genes, and SYBR green as a fluorescing agent, two real-time PCRs were developed. Testing diluted DNA samples showed that the mosaic penA gene PCR was 10-100 fold more sensitive than the wild type gene PCR. Both PCRs were negative with strains belonging to N.meningitidis $(\mathrm{n}=3), N$.lactamica $(\mathrm{n}=4), N$.subflava $(\mathrm{n}=2)$, N.cinerea $(\mathrm{n}=1)$ and N.elongata $(\mathrm{n}=1)$. Ten urine $(\mathrm{U}), 10$ cervical $(\mathrm{C}), 10 \mathrm{rectal}(\mathrm{R})$ and 10 tonsillar ( $\mathrm{T}$ ) samples, all negative in the NAAT for $\mathrm{Ng}$, were negative in both PCRs. Testing paired samples from patients, who had a positive culture and NAAT (10 R, $9 \mathrm{U}, 8 \mathrm{C}, 9 \mathrm{~T})$ showed concordant results in 35/36 samples: 4 pairs tested positive in the mosaic PCR and 31 in the wild type PCR. From one patient a wild type strain had been cultured from the throat, but both PenA PCRs on the swab were negative, possibly due to a low amount of DNA.

Conclusion We successfully developed discriminating PCRs with which the $\mathrm{Ng}$ mosaic penA gene can be detected without culture of $\mathrm{Ng}$. This test can be used to estimate the prevalence of diminished susceptibility of $\mathrm{Ng}$ against cephalosporins in regions where culture is no longer performed.

\section{P2.098 SPECIATION AND ANTIFUNGAL SUSCEPTIBILITY TESTING OF CANDIDA SPECIES CAUSING ORAL THRUSH IN HIV PATIENTS}

doi:10.1136/sextrans-2013-051184.0362

S Shreedhar, ${ }^{2} \mathrm{~V}$ K Saralaya. 'Final year Undergraduate Student of Medicine, Kasturba Medical College (Affiliated to Manipal University), Mangalore, India; ${ }^{2}$ Dept. of Microbilogy, Kasturba Medical College (Affiliated to Manipal University), Mangalore, India

Purpose Oral thrush by Candida species is a common ailment of individuals suffering from HIV infection. These species show a high resistance to antifungal drugs used for the treatment. Hence our study was conducted to determine the aetiology and antifungal susceptibility patterns of Candida isolates causing oral thrush in HIV patients.

Materials and Methods: Isolation of Candida species was attempted from 60 cases of oral thrush in HIV infected patients at the Department of Microbiology, Kasturba Medical College, Mangalore. Isolates were identified to species level based on chlamydospore formation; ability to form germ tube; assimilation/ fermentation of carbohydrates; production of urease enzyme; formation of pellicle/surface film on Sabouraud's dextrose broth; growth on Sabouraud's Dextrose Agar (SDA) with cycloheximide and growth on SDA at 370C and 450C. Antifungal drug susceptibility testing was done by macro broth dilution test using azole group such as fluconazole, itraconazole and ketoconazole.

Results 56 Candida species were isolated of which C.albicans was the predominant isolate (84\%), followed by C.tropicalis (8\%), C.glabrata (3.5\%), C.parapsilosis and C.kefyr (1.8\% each). Most isolates (53) showed significantly higher resistance to fluconazole than the standard pathogenic control strain C.albicans NCPF 3153A. 31 isolates $(66 \%)$ of C.albicans had Minimum Inhibitory Concentration (MIC) values 8 times that of control for ketoconazole. 23 isolates had MIC for itraconazole of $0.5 \mu \mathrm{g} / \mathrm{ml}$ which was only twice as high as that of control $(0.125 \mu \mathrm{g} / \mathrm{ml})$, all others having comparatively equivalent MIC to itraconazole.

Conclusion Our study indicates that although C.albicans is the predominant species, there are other species prevalent and causing infection in our HIV infected population. MIC's of our Candida isolates to commonly used antifungals such as fluconazole, ketoconazole and itraconazole were significantly higher than the control strain used in the study. Our study indicated that itraconazole was the most effective among the azole group of drugs.

\section{P2.099 VEHICLE ALTERATIONS IN PODOPHYLOTOXINE TREATMENT: A PARTIAL DISAPPOINTMENT}

doi:10.1136/sextrans-2013-051184.0363

'F V Ser, ${ }^{2} \mathrm{~N}$ Raketic, 'S Zoric, ${ }^{3} \mathrm{Z}$ Zoric. ${ }^{1}$ City Departement for Skin and Venereal Diseases, Belgrade, Serbia, ${ }^{2}$ Ordinacija Raketic, Belgrade, Serbia, ${ }^{3} \mathrm{DZ}$ Milutin Ivkovic, Palilula, Belgrade, Serbia

Background Podophylotoxine o, o5\% gel is a routinely used for condylolma accuminatum treatment as home used procedure. However, irritations, sometimes severe are common. This may be due partially to leakage of the preparation to the surrounding noninfected tissue. It is difficult to expect the preparation to remain dry and only on affected areas in non-circumcised males and females.

Methods :We used compounded podophylotoxin o, 15\% in adhesive creamy base. The team work with pharmaceutical technologist helped to create an adhesive creamy paste, with greater stability and uniformly distribution on individual lesions. The melting of the preparations was minimised by tailored compounding.

Results The vast majority of patients preferred cream to gel, both to far less irritations and excellent tolerability. Unfortunately, the overall success with cream formulation was disappointing. Therapeutic results seemed to be better, at the very beginning of the treatment, dye to the constant and prolonged delivery on the treated lesions. However, recurrences are far more frequent and tend to develop earlier than with gel podophylotoxin formulation.

Conclusion Probably, the cream formulation does have a therapeutic advantage in perianal region, because of the better adherence of the vehicle, and, when in out of office settings, of less irritation to surrounding tissue in the presence of over-applying the medicine, which frequently is the case.

\section{P2.100 CLINICAL EFFICACY OF SITAFLOXACIN 100MG TWICE DAILY FOR 7 DAYS FOR PATIENTS WITH NON- GONOCOCCAL URETHRITIS}

doi:10.1136/sextrans-2013-051184.0364

${ }^{1,2}$ S Takahashi, ${ }^{2} \mathrm{R}$ Hamasuna, ${ }^{2} \mathrm{M}$ Yasuda, ${ }^{2} \mathrm{~S}$ Ito, ${ }^{2} \mathrm{~T}$ Deguchi, ${ }^{2} \mathrm{~T}$ Matsumoto. ${ }^{1}$ Department of Urology, Sapporo Medical University, Sapporo, Japan; 'Japanese Research Group for UTI, Kitakyushu, Japan

To date, the standard treatment for the patients with chlamydial non-gonococcal urethritis (NGU) remains effective; however, conventional quinolone antibiotics have less activity against Mycoplasma 